\title{
CORRECTION OF THE PRONATED FOOT
}

\author{
G. K. Rose, Shrewsbury, ENGLAND \\ Consultant Orthopaedic Surgeon to the Robert Jones and Agnes Hunt Orthopaedic Hospital \\ and the Royal Salop Infirmary
}

Traditionally, correction of the pronated foot has been achieved by the use of a raising applied to the inner border of the heel or the heel and sole of the shoe, this raising being applied either to the under surface of the sole or, less commonly, to the inside of the shoe. In addition to, or in place of, this method various designs of insoles have been used, or even larger appliances such as an outside iron and an inside T-strap. In considering the relative efficiency of these measures it is important to have some understanding of the mechanics involved, particularly of the peritalar (talo-calcaneo-navicular) joint complex, and also of another joint not previously described, namely the calcaneo-contact articulation.

\section{MECHANICS OF THE FOOT}

The simplest type of joint used in everyday life is that of a hinge. The axis of such a joint is clearly the pin running down the centre of the hinge, for it is about the line of this pin that the parts constituting the joint rotate (Fig. 1). If such a hinge is modified, as shown in Figure 2, the axis becomes an imaginary line, and this is a mathematical expression of the shape of the joint surfaces which must be part of the circumference of a circle drawn around the axis-or, more accurately, where there is a single axis to a joint, lines bisecting any chord of the joint surface at right angles in one plane will pass through the same spot. This seemingly pedantic correction is of real importance in considering the function of anatomical joints, for it must be always borne in mind that, no matter how accurately an axis may have been determined experimentally, it will in a particular case depend on the integrity of the joint surfaces, and its ligaments. Provided these conditions are fulfilled its surfaces may be segmented, and special forms of segmental joints of this type can be constructed in which the segments are situated on the opposite sides of the axis (Fig. 3). Furthermore, it should be noted that the radius of each segment need not be the same, provided the one axis is used. A well known example is the articulation of the radius and ulna, where the segments are widely separated, but nevertheless essentially integrated components of a single joint.

No apology is made for the reiteration of first principles, for if these axes are to become the tools of the clinician their uses and limitations must be clearly understood.

The analogy of the hinge can easily be translated into anatomical terms-for example, the interphalangeal joints of a digit. Many joints, the ball-and-socket type in particular, have no such simple axis; but fortunately for the purpose of this paper, as will be seen later, they need not complicate our thinking.

The determination of the axes of the joints of the foot has proved a fascinating source of interest for well over fifty years. Fick (1911), referring to the talo-calcaneo-navicular joint complex, stated that the movement was a simple hinge movement, and that the whole of the subtalar portion of the foot rotated about a line passing obliquely through the head of the talus. Manter (1941), Shephard (1951) and Hicks (1951) have been among the more significant contributors, the work of Hicks being most comprehensive and satisfying so far. He has shown that in the normal foot all movements and changes in the shape of the foot are the result of rotations about axes, and he has determined these axes for each joint. His results correspond closely to those of Manter and Shephard in regard to the axis of what Shephard named the peritalar joint, or the talo-calcaneo-navicular complex. The position of this can 
be seen in Figure 4, and Shephard showed that the axis of the talo-navicular joint, as determined from the joint surfaces, also corresponds to the axis of the subtalar joint.

It is a property of hinge joints that they are what Steindler has called " trackbound ": that is to say the plane of movement of a joint is always perpendicular to its axis, and different

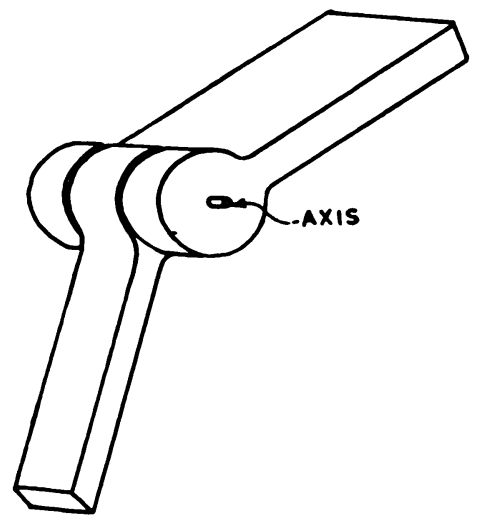

Fig. 1

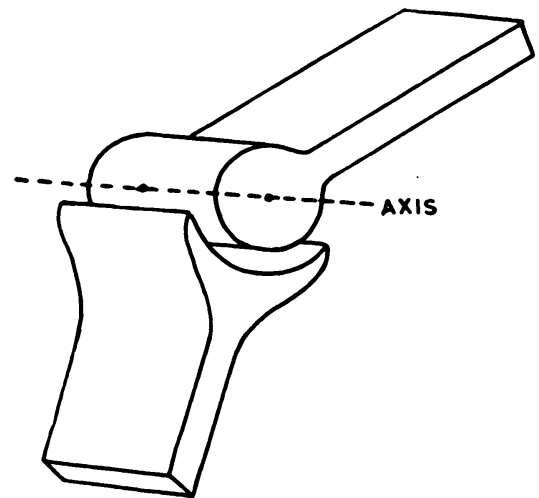

Fig. 2

Figure 1-Solid axis of a hinge. Figure 2-Modification of the joint to conform to an anatomical hinge joint. The axis now becomes a mathematical expression of the curvature of the joint surfaces.
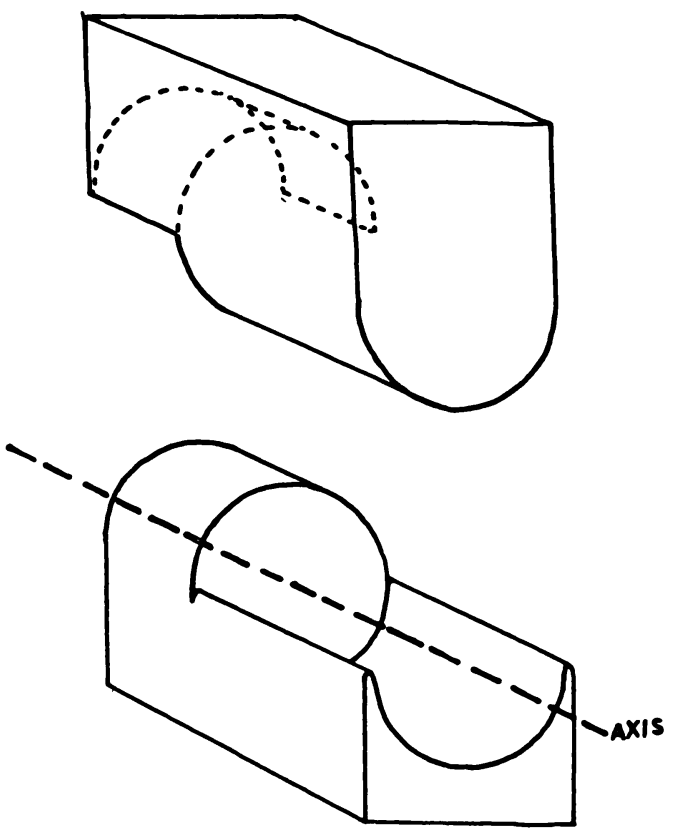

Fig. 3

A modified anatomical hinge joint with segmented articulations on the opposite sides of a single axis.

This joint corresponds closely to the subtalar joint.

forces applied to this joint, although they may run in oblique direction, always produce one of two movements, a clockwise or anti-clockwise rotation about the axis-quite unlike the movement produced at a ball-and-socket joint, which will always be in the plane of the resultant of forces acting upon the joint. In the hinge joint the efficiency of the movement compared with the force will be most marked when the force is at right angles to the axis, 
diminishing to nil when it lies in the line of the axis. Physiology must not be forgotten in considering this mechanical situation, and it is clear that the trackbound quality of joints has to be maintained by a certain balance of muscles acting about a joint. Consider for example the function of the saddle-shaped trapezio-metacarpal joint. It is a common experience that when muscle transplants are performed to produce opposition of the thumb in a paralysed hand they are usually successful at first, but fail as the trackbound quality of the joint becomes lost under the repeated stress of a tension pulling in a single plane; then the thumb, instead of performing a movement closely resembling normal opposition, merely adducts across the palm.

That the movement of all joints in the foot-and in particular the movement of the peritalar joint complex -is a pure rotation, has been disputed by many observers who have noticed a gliding movement in the mid-tarsal region, and this they have attributed to a glide occurring at the subtalar joint. In fact, however, it is produced at the calcaneo-contact

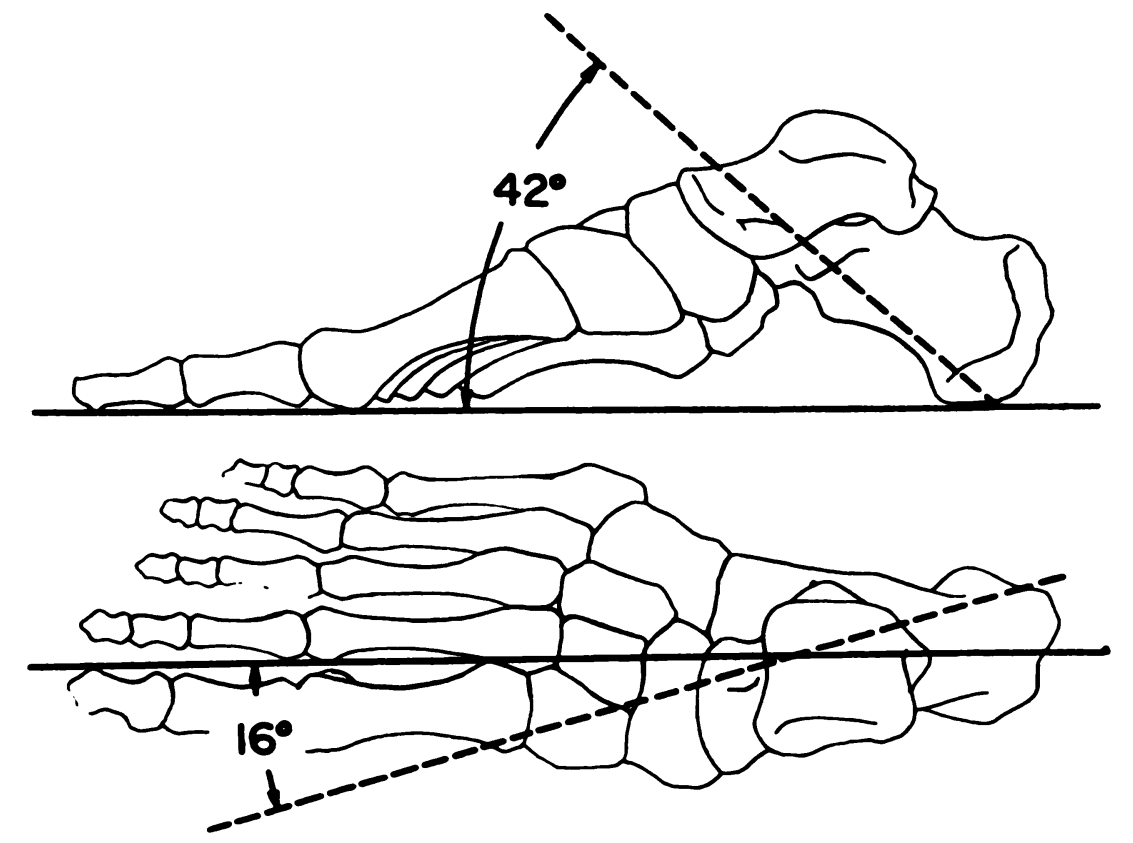

Fig. 4

To show the axis of the subtalar joint. (From Manter 1941.)

articulation. It has long been recognised that the movements of the foot appear different in the standing foot from those that are observed when the foot is held above the ground, and it will be seen that this difference is the product of a particular movement occurring at the calcaneo-contact surface and the rotation at the peritalar axis.

The weight-bearing surface of the calcaneum is dome-shaped. In the antero-posterior plane, part of this dome has an important function in the heel-and-toe action of normal walking; but when the foot is used as a static weight-supporting structure it is the transverse contact area of this dome which becomes important, and when the foot is fully weight bearing a modified type of trackbound articulation is formed between the calcaneum and the ground, or, more accurately, in part between the skin beneath the calcaneum and the ground, and in part between the calcaneum and the fibro-fatty pad between it and the skin. This articulation differs from the joints of the foot in that it is not a simple hinge joint, but has the same mechanism as does that of a wheel moving over the ground, the axis itself moving in space parallel to the ground as rotation occurs around it. In other words the surfaces do not slide 
one over the other, lacking the lubrication and frictional qualities of articular cartilage, but roll one on the other with a high degree of friction between them (Fig. 5).

Since for practical purposes the axis of the subtalar joint may be considered to be embedded in the calcaneum, it will be seen that this causes a movement in space of the subtalar axis about which rotation of the talus can occur relative to the calcaneum (Fig. 7).

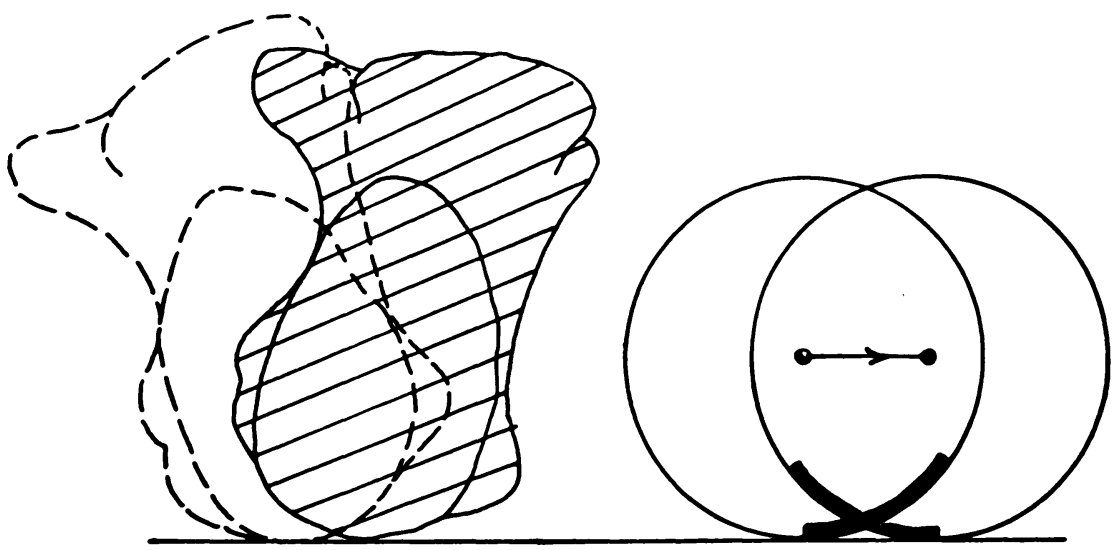

Fig. 5

Calcaneo-contact articulation showing the wheel-like movement.

Furthermore, in the standing subject with the knees straight any movements of the talus which occur are, again for practical purposes, linked through a solid rod comprising the tibia and the femur to the hip joint, which in the conditions laid down will allow rotation only about a vertical axis.

In order to translate this into visual terms a model was constructed of acrylic, the subtalar joint being represented by two plain surfaces separated by a metal spacer, the axis of the

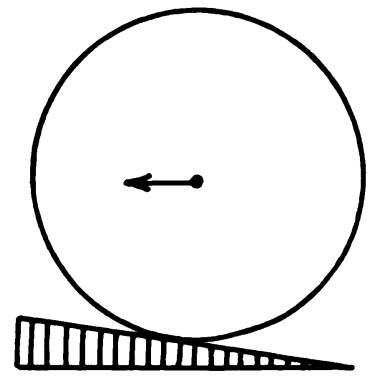

$x$

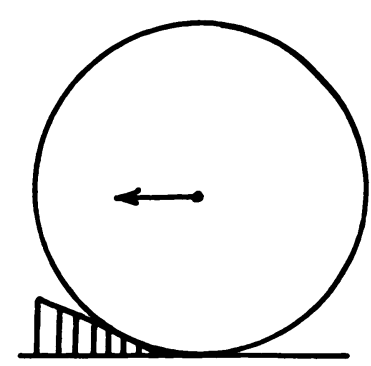

Y

Fig. 6

To demonstrate the efficiency of a wedge placed medial to the calcaneum as opposed to that of a generalised raise of the inside of the heel in preventing inward roll. $Y$ is much more effective than X.

joint being embedded in the calcaneum and running freely through the talus. The tibia and femur were represented by a single bar embedded into the upper surface of the talus, and this allowed to rotate in a loose ring at the top of the stand (Figs. 8 to 10). From this model certain important practical points immediately became obvious. 1) The wheel effect of the calcaneo-contact joint is shown (Figs. 8 and 9). 2) This is accompanied by a shift in the 
peritalar axis embedded in the calcaneum which, interreacting with the rotation possible in the bar representing the locked tibia and femur, produces a movement of the head of the talus which is in the almost horizontal plane, trackbound through the rotation of the tibia and the roll of the calcaneum at the calcaneo-contact articulation. The trackbound quality of this linkage is emphasised by the fact that only movement of the head of the talus in a clockwise or anti-clockwise direction relative to the tibial axis can be produced, no matter in what direction force is applied to the linkage. Conversely, as the foot pronates, the head

the talus moves inwards and exerts the greatest force in a horizontal plane against the 'ateral wall of the peritalar joint.

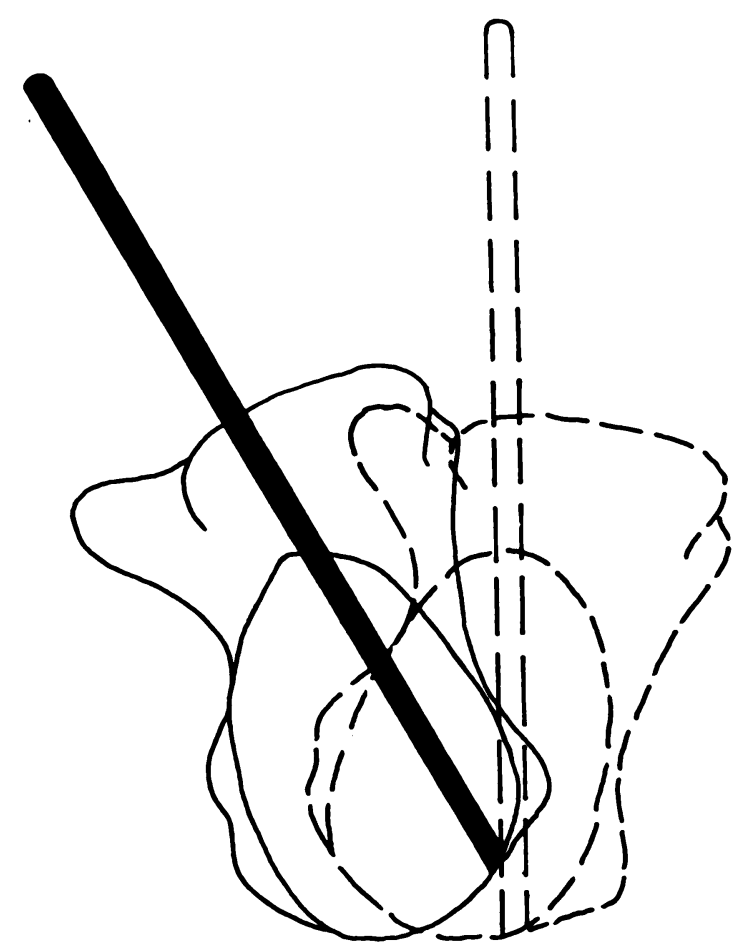

Fig. 7

The glide in space of the axis of a peritalar joint with movement of the calcaneo-contact joint.

On this model it is quite clear that pressure from below the talus and vertically upwards achieves nothing, except a tendency to lift the whole foot upwards, whereas the most efficient correction of the in-turned talar head is relatively easily achieved when a horizontal force is applied from the inner border of the foot outwards in the coronal plane against the head of the talus itself. This clearly demonstrates the futility of the internal longitudinal arch support as a corrective device and relegates it to its proper position as a simple support of osteoarthritic joints. Because of the trackbound effect already noted this correction can also be brought about by twisting the calcaneum or the tibia; but clinically these methods are much less practical. These points were fully appreciated by Whitman when he designed his plates, and this principle of correction was advocated later by Charlesworth and more recently by Helfet, who has produced a more modern and effective version of the Whitman insole.

Elevation of the inner border of the shoe acts in two ways. 1) By tilting the calcaneum. Its effectiveness in this direction is limited by the fact that the raise of the medial border both lifts and tilts the calcaneum, so that the amount of correction that can be produced is 


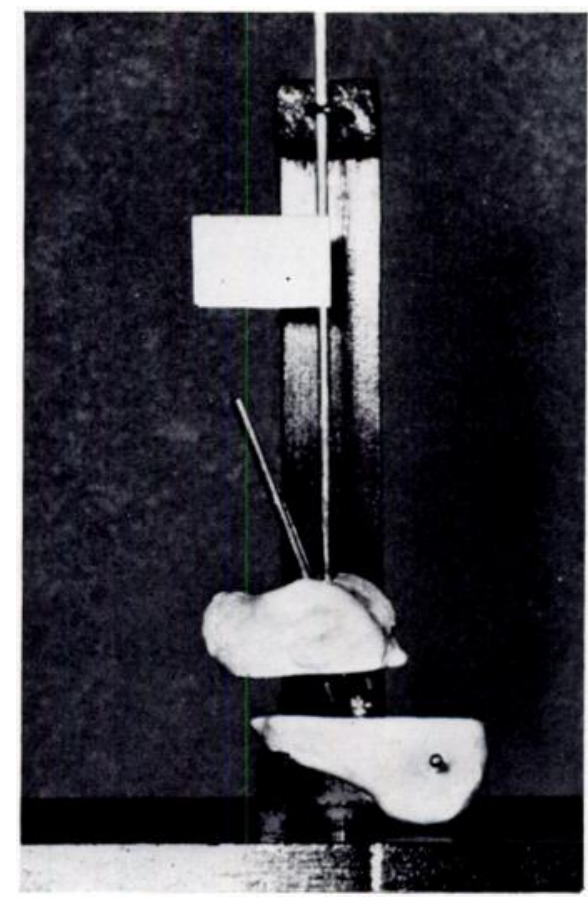

FIG. 8

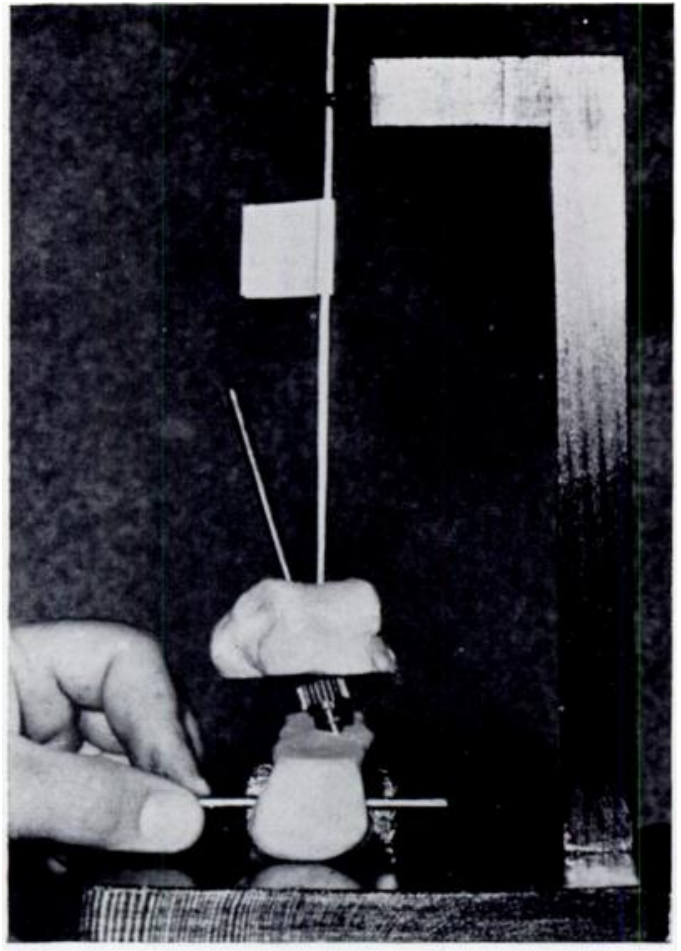

Fig. 9

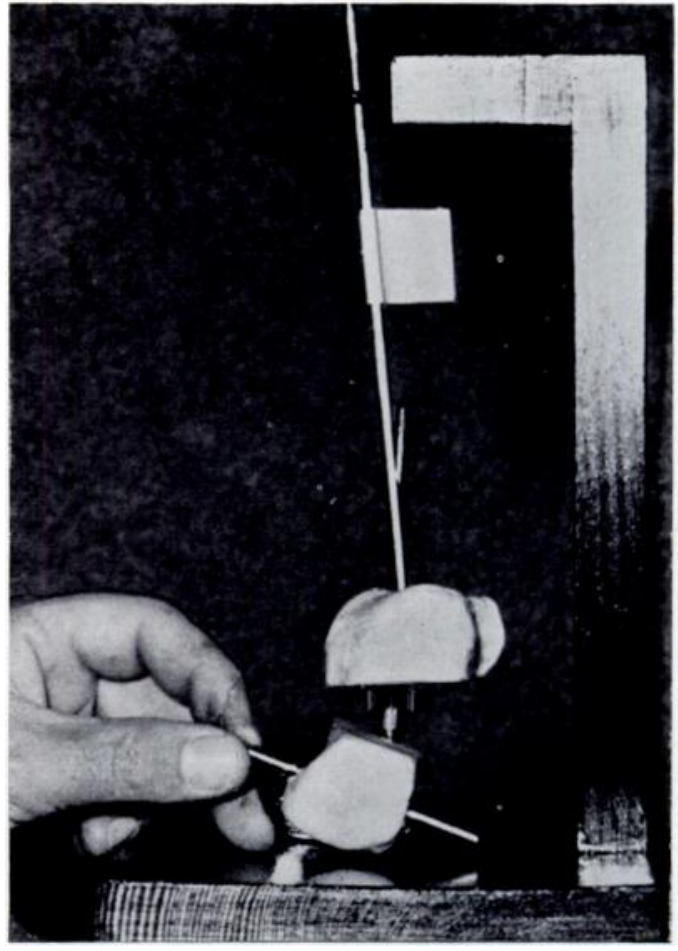

Fig. 10

An acrylic model to demonstrate the trackbound relationship between the tibia, the peritalar joint axis and the calcaneo-contact joint in the standing weight-bearing foot.

VOL. $40 \mathrm{~B}$, NO. 4, NOVEMBER 1958 
ultimately limited by the angle of slope of the heel. If this becomes too great, it causes the foot to slide laterally and lie in an uncorrected position, between the angle of the heel and the outer wall of the upper. This correction is effective whether the raise is applied to the outside of the shoe or to the inside. The degree of correction provided by this method alone

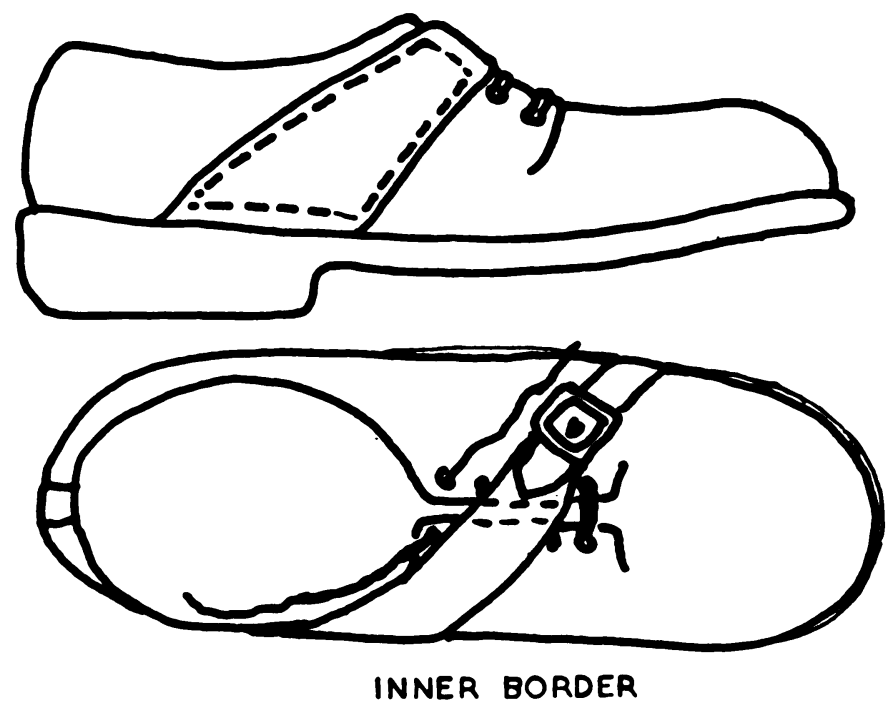

Fig. 11

Valgus strap to reinforce the pressure over the inner side of the talar head. The dotted lines in the upper figure indicate a stiffening inserted into the strap.

is relatively small. Schwartz has pointed out that a wedge inserted inside the shoe will have a different effect from a generalised raise of the inside of the heel, and can be used to prevent inward rolling of the calcaneum at the calcaneo-contact articulation. It has the disadvantage that the degree of correction is not easy to control (Fig. 6). 2) By the pressure of the inner

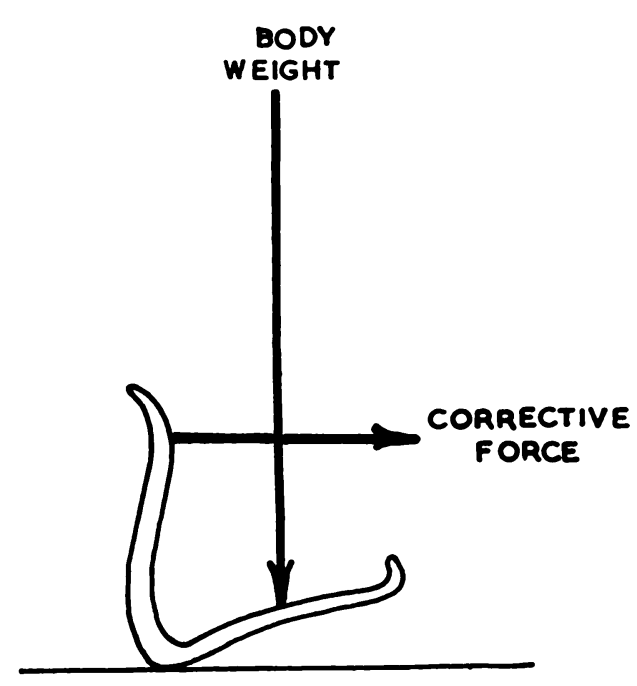

Fig. 12

Illustrates the transformation of the vertical force of body weight into a corrective horizontal force by means of the heel cup. This is a section through the cup. border of the shoe in an outward direction on the region of the head of the talus. This is in turn limited by the rigidity of the upper of the shoe in the region of pressure, and it is common to see that this rigidity is inadequate and the leather is deformed here. In practice this is probably the most effective action of a medial raise, and it occurs much less efficiently when the raise is placed inside the shoe rather than on the outer surface of the heel. When a correction of only a modest degree is required, this method of treatment may be reinforced by the addition of a valgus strap (Fig. 11). In more severe cases the type of heel cups described by Helfet (1956) are extremely good. They provide a means of transmitting the vertical body weight into a horizontal force (Fig. 12). Their effectiveness depends upon: 1) the manufacture of the insoles with the foot fully corrected; and 2) the manufacture of the insoles with materials that will neither deform nor crack under the considerable force applied. 


\section{CONSTRUCTION OF INSOLES}

Taking the pattern-A cast of the foot is made either in putty or preferably with a plaster slab, the foot weight bearing, the correction being achieved by full dorsiflexion of the great toe, assisted, if necessary, by lateral rotation of the tibia. Many instrument makers seem to

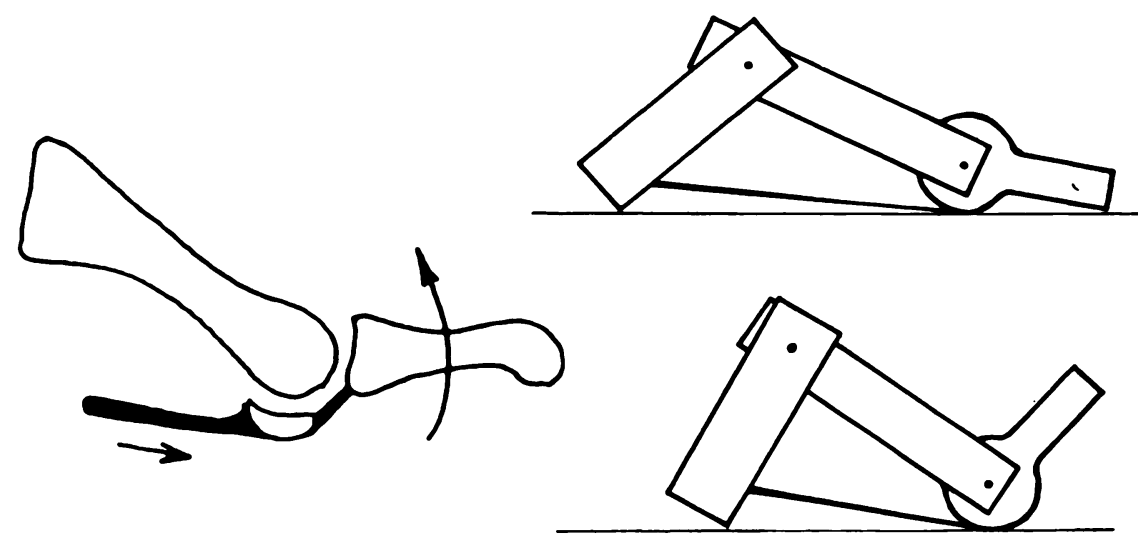

Fig. 13

Illustrates (left) the anterior attachrnent of the plantar aponeurosis to the proximal phalanx of the great toe through the sesamoids and (right) the windlass effect on extending the great toe. (After Hicks, 1951.)

be reluctant to make an insole from a weight-bearing case which, with its flattened heel surface, has a marked difference in appearance from the traditional insole. Nevertheless if pressure areas on the inner side of the foot are to be avoided it is essential that the insole be made to fit the weight-bearing foot.

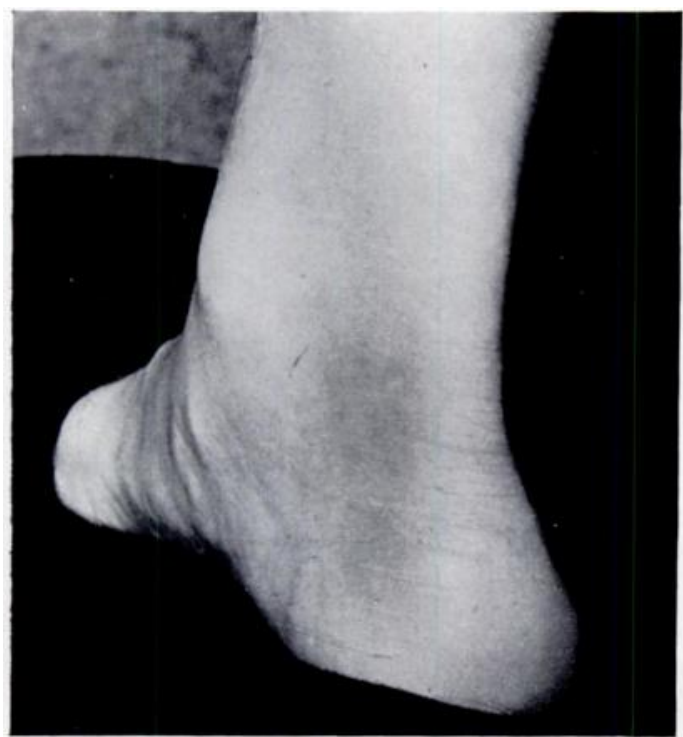

FIG. 14

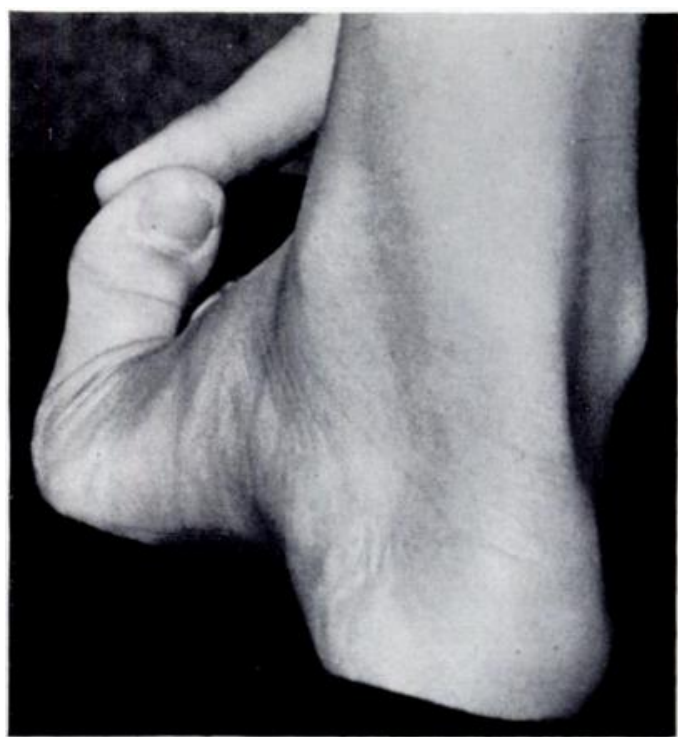

Fig. 15

Photographs illustrating the rise in the medial longitudinal arch and the roll of the calcaneum that accompany extension of the great toe owing to the windlass effect of the plantar aponeurosis.

Correction of the foot deformity by dorsiflexing the great toe was described by Jack (1953), and the mechanism by which it is achieved was elucidated by Hicks (1951). Hicks showed that the plantar aponeurosis, attached behind to the calcaneum and in front in the case of 
the great toe to the base of the proximal phalanx through the sesamoid bones, acts as the rope of a windlass, the metatarsal head being the drum and the proximal phalanx the handle. Raising the great toe increases the tension in the fascia and also causes a shortening relative to the internal longitudinal arch of the foot. In consequence the calcaneum is rolled outwards with the effects that have already been made clear from the model, and the first metatarsal ray is flexed, which in the standing foot also elevates the arch (Figs. 13 to 15). This is an important observation which can be put to practical use in the taking of impressions from the foot.
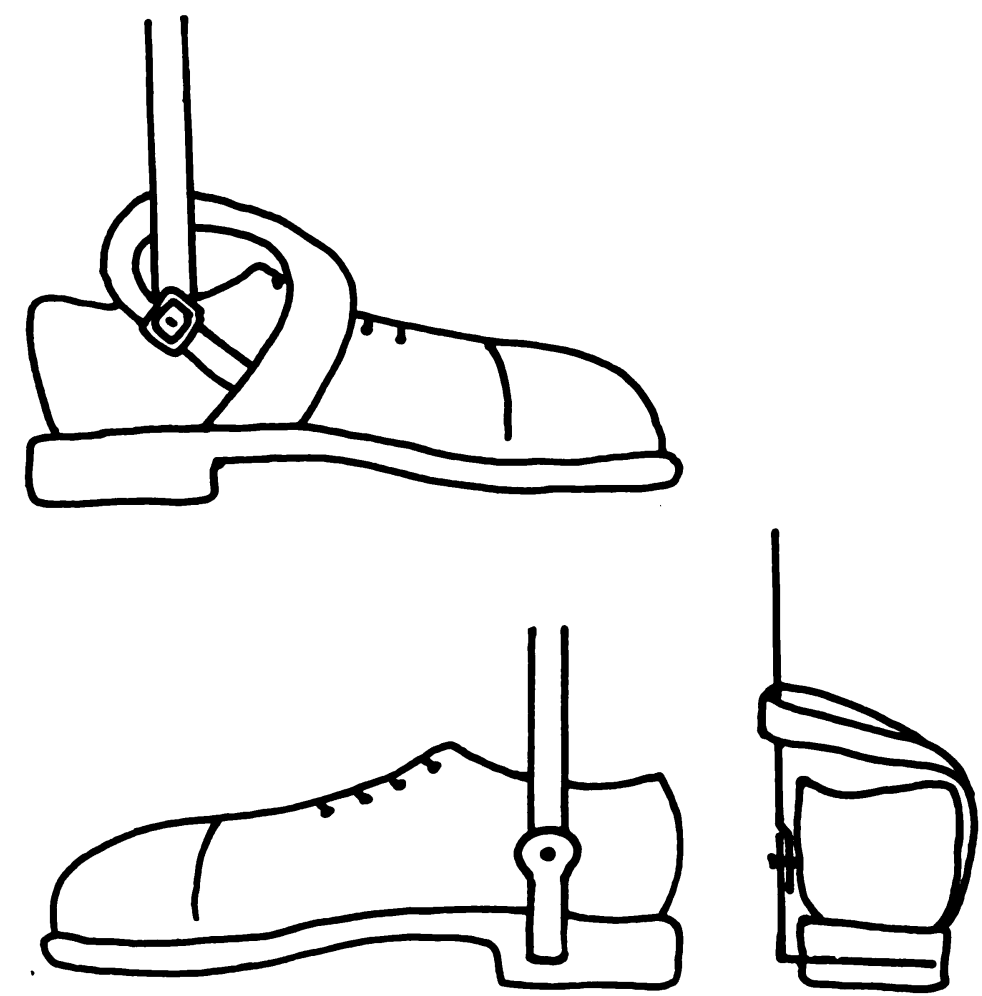

Fig. 16

Special modification of the outside iron and inside T-strap. It will be noted that the T-strap is modified to bring the maximum pressure over the talar head, and as this instrument is used in small children very frequently, it has been found that the ordinary socket into the heel is technically difficult and heavy, so that a light simple hinge has been used, as indicated.

Materials used-Durability of the arch supports is best achieved by manufacture with laminations of ordinary surgical bandage impregnated with a cellulose cement, reinforced with what in the boot trade is called " bottom filler," which is powdered cork impregnated in the cellulose cement (Charlesworth 1951). In prolonged use these have proved extremely satisfactory. Block-leather heel cups easily go out of shape under the influence of sweating, and fibre-glass or other plastics tend to crack.

\section{COMMENTS}

These heel cups cannot be used for small children, in whom the most efficient type of corrective device is the outside iron with an inside T-strap. Special care should be taken over the design of the T-strap in order that the entire force of this strap should be a horizontal transverse force on the head of the talus. Figure 16 shows the important difference between the traditional strap and the one which is recommended.

THE JOURNAL OF BONE AND JOINT SURGERY 
No special consideration has been given here to the difference in effectiveness between an inside raise of the heel only, often prolonged forward for strength, and an inside raise of the sole and heel. This question cannot be considered from a theoretical point of view without bringing in further considerations regarding the mechanics of the forefoot and the interrelation between these and the hind foot which have not been dealt with in this paper. Nevertheless it is clear from the arguments adduced here that an inside raise of the sole and heel of the shoe will tend to produce a better horizontal corrective force against the talus head than a raise only of the heel, and this in my opinion is sufficient to make this prescription universal. There are further good reasons to support this view from considerations of the mechanics of the foot as a whole, and these will be dealt with later.

\section{SUMMARY}

1. The mechanical qualities of the peritalar joint, the calcaneo-contact joint, and their interreaction with the hip joint in the standing weight-bearing foot are considered. 2. The most efficient ways of correcting the pronated foot have been indicated.

I am indebted to the Research Committee of the Birmingham Regional Hospital Board for a grant which made this work possible.

\section{REFERENCES}

Charlesworth, F. (1951): Chiropodial Orthopaedics, p. 108. Edinburgh and London: E. \& S. Livingstone Ltd. FICK, R. A. (1911): Handbuch der Anatomie und Mechanik der Gelenke unter Berücksichtigung der bewegenden Muskeln. Jena: G. Fischer.

Helfet, A. J. (1956): A New Way of Treating Flat Feet in Children. Lancet, i, 262.

Hicks, J. H. (1951): The Function of the Plantar Aponeurosis. Journal of Anatomy, London, 85, 414.

Hicks, J. H. (1953): The Mechanics of the Foot. I. The Joints. Journal of Anatomy, 87, 343.

Hicks, J. H. (1954): The Mechanics of the Foot. II. The Plantar Aponeurosis and the Arch. Journal of Anatomy, 88, 25.

Hicks, J. H. (1955): The Foot as a Support. Acta Anatomica, 25, 34.

Inman, V. T., and Close, J. R. (1953): The Action of the Subtalar Joint, Advisory Committee on Artificial Limbs National Research Council, Prosthetic Devices Research Project. Institute of Engineering Research, University of California, Series II, Issue 24.

JACK, E. A. (1953): Naviculo-cuneiform Fusion in the Treatment of Flat Foot. Journal of Bone and Joint Surgery, 35-B, 75.

JoNES, F. Wood (1946): Structure and Function as Seen in the Foot. London: Baillière, Tindall and Cox.

Levens, A. S., Inman, V. T., and Blosser, J. A. (1948): Transverse Rotation of the Segments of the Lower Extremity in Locomotion. Journal of Bone and Joint Surgery, 30-A, 859.

MaNTER, J. T. (1941): Movements of the Subtalar and Transverse Tarsal Joints. Anatomical Record, 80, 397. Saunders, J. B. DeC. M., Inman, V. T., and Eberhart, H. D. (1953): The Major Determinants in Normal and Pathological Gait. Journal of Bone and Joint Surgery, 35-A, 543.

Schwartz, R. P. (1958): Personal communication.

SHEPHARD, E. (1951): Tarsal Movements. Journal of Bone and Joint Surgery, 33-B, 258.

Steindler, A. (1955): Kinesiology of the Human Body. Springfield, Illinois: Charles C. Thomas.

Whitman, R. (1888): Observations of Forty-five Cases of Flat-foot with Particular Reference to Etiology and Treatment. Boston Medical and Surgical Journal, 118, 598; 616.

Whitman, R. (1946): A Review of the Inception and Development of a Type of Reparative Surgery Adapted to Bodily Mechanics. Journal of Bone and Joint Surgery, 28, 374.

WILES, P. (1934): Flat-Feet. Lancet, ii, 1,089.

Wiles, P. (1949): Essentials of Orthopaedics. London: J. \& A. Churchill Ltd. 